\title{
Achievable Capacity Design for Irregular and Clustered High Performance Mesh Networks
}

\author{
Thomas Otieno Olwal ${ }^{1}$ and Moshe Timothy Masonta ${ }^{1,2}$ \\ 1 Council for Scientific and Industrial Research (CSIR), Meraka Institute, P. O. Box \\ 395, Pretoria, 0001, South Africa, \\ \{tolwal, mmasonta\}@csir.co.za, \\ 2 Department of Electrical Engineering, Tshwane University of Technology, Private \\ Bag X680, Pretoria, 0001, South Africa
}

\begin{abstract}
This study has presented the End to End (E2E) upper bound capacity limits for high performance mesh nodes that can be deployed in rural and remote areas. The achievable capacity limits for both irregular and clustered placements of nodes have been analytically derived. Numerical results based on the data sheets of IEEE $802.11 \mathrm{a} / \mathrm{n}$ standards reveal the efficacy of such designs to typical rural networks such as the Peebles valley mesh in rural South Africa.
\end{abstract}

Key words: Achievable capacity, clustered \& irregular placement, HPN

\section{Introduction}

The next generation fixed wireless broadband networks have increasingly been deployed as mesh networks in order to provide and extend access to the internet. These networks are characterized by the use of multiple orthogonal channels available within the industrial, scientific and medical (ISM) licensedfree frequency bands. Nodes in the network have the ability to simultaneously communicate with many neighbors or stream different versions of the same data/information using multiple radio devices over orthogonal channels thereby improving effective "online" channel utilization [1]. The ability to perform full duplex communication by individual multi-radio nodes without causing network interference has also been achieved through decentralized transmission power control schemes in [2], [3]. That is, one radio interface can be used for receiving packets and the other radio interfaces on seperate non overlapping channels are used for transmitting packets. Many such networks emerging from standards such as IEEE $802.11 \mathrm{a} / \mathrm{b} / \mathrm{g} / \mathrm{n}$ and 802.16 are already in use, ranging from prototype test-beds [4] to complete solutions [5].

The increasing question is how the end to end (E2E) theoretical capacity of such multi-radio network scales with the node density, irregularity of node placement and link obstructions [6]. In their seminal work, Gupta and Kumar [7] determined the capacity of single radio single channel networks. Their findings have been later extended to derive the capacity bounds of a high performance network [8]. In addition, the link throughput performance parameters in IEEE 
802.11 networks have also been discussed in [9]. However, the considered network architectures have so far been presented with a number of impractical assumptions. The first assumption asserts that the location of nodes and traffic patterns can be controlled in arbitrary networks. The second assumption claims that channel fading can be excluded in the capacity analysis such that each frequency channel can support a fixed data rate. Lastly, nodes are randomly located on the surface of a torus of unit area to avoid technicalities arising out of edge effects. However, in realistic networks, location of nodes is determined by the irregularity of the terrain, the presence of tree foliage [6], and needs and locations of terminal users [10]. Moreover, typical rural based wireless networks can be described by (i) long single hop links, (ii) limited and unreliable energy sources, and (iii) clustered distribution of Internet users [11]. The main problem constitutes the need to increase capacity of community owned existing wireless broadband networks so that multimedia services can be delivered to remote and rural areas without losing connectivity [2].

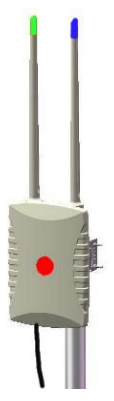

Fig. 1: High Performance Node $(H P N)^{T M}[10]$

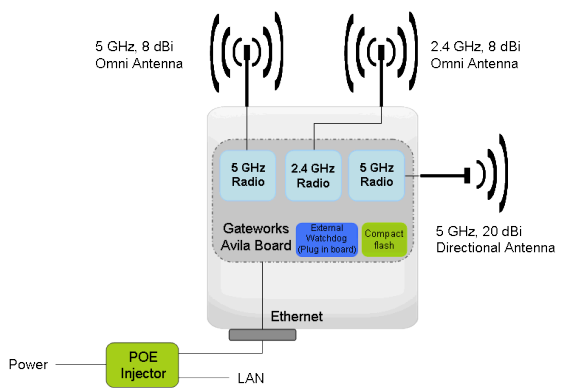

Fig. 2: Block Diagram of HPN ${ }^{\mathrm{TM}}$

In response to this need, high performance nodes (HPNs) ${ }^{\mathrm{TM}}$ for communityowned wireless mesh networks, have been implemented in most parts of rural South Africa [12]. The innovation as shown in Figure $\mathbf{1}$ has been developed by the CSIR Meraka Institute and it provides high throughput in mesh networks. The HPN ${ }^{\mathrm{TM}}$ is an IEEE 802.11 based multi-interface node made up of three interfaces or radio devices and controlled by an embedded microcontroller technology [10]. To ensure high speed performance, the innovation has the first radio interface card attached to a $5 \mathrm{GHz}$ directional antenna for backhaul mesh routing; the second interface card is connected to a $5 \mathrm{GHz}$ omni-directional antenna for backhaul mesh connectivity and access. The third radio interface card is attached to a $2.4 \mathrm{GHz}$ omni-directional antenna for mesh client access network. As shown in Figure 2, the HPN block diagram has a weather proof Unshielded Twisted Pair (UTP) connector at the bottom of the node that provides PowerOver-Ethernet (PoE) and Ethernet connectivity to the HPN. To attach the HPN to a pole or a suitable structure, a mounting bracket is fixed at the back of the 
router for other operational details [10]. The HPNs are often installed on roof tops, street poles and buildings of villages, local schools, clinics, museums and agricultural farmlands.

In this study, the focus will be the design of E2E capacity limit of the terminal backhaul connectivity of the HPNs. The terminal backhaul connectivity offers aggregated traffic volumes of all flows within the network. The traffic flows traverse long links between any two HPNs and are faced with severe climatic and topographical conditions [11], [12]. Thus, E2E upper bound capacity limits for irregular and clustered placement of HPNs are analytically found to be as follows:

$$
O\left(R n \sqrt{\frac{m c}{\delta p}}\right) \text { and } O\left(R n \sqrt{m c\left[\frac{n_{1}}{\delta_{1}}+\frac{n_{2}}{\delta_{2}}\right]}\right) .
$$

Here, $R$ is the rate of a single link in bits/s computed by taking into account multipath effects and the built-in structure of the innovative HPNs, $n$ is the number of HPNs, $m$ is the number of radio interface cards per each HPN, $c$ is the number of frequency channels that do not cause interference in duplex communication, $0<p<1$ is the rate of the regularity (probability) of the placement of HPNs, and is the HPN distribution density that is varied over a fixed deployment area. In clustered placements, $\delta_{1}$ and $n_{1}$ are the intra-cluster density and number of HPNs, respectively. On the other hand $\delta_{2}$ and $n_{2}$ are similar values for the inter-cluster.

The rest of the paper is organized as follows. In Section 2, design of upper bounds E2E capacity limits for HPN networks is provided. Section 3 furnishes numerical capacity limits and related discussions for real networks in a given rural area size. The paper is concluded in Section 4, with highlights of the main contributions of this study, and future research and development (R\&D) perspectives.

\section{Achievable capacity of HPN wireless mesh}

In order to design the achievable capacity bound for the HPN (the dual channel dual radio) based mesh network we consider a typical static wireless mesh network. Suppose the network is assumed to consist of varying $n$ number of HPNs within a fixed area of deployment region (i.e., $5 \mathrm{Km}$ by $5 \mathrm{Km}$ ). Also to generalize our derivations and only apply specific cases later with numerical examples, we employ the approach presented by [8] in order to investigate the impact of number of channels and interfaces on the capacity of multi-channel wireless networks. In our derivations, the term "channel" will refer to a part of frequency spectrum with some specified bandwidth and the term "radio" will mean the network interface card. Let us assume that the HPNs based mesh network has channels and every node is equipped with $m$ interfaces so that the relation between the number of interface cards and channels is $2 \leq m \leq c$. Each interface card can only transmit and receive data on any one channel at a given time. It is a half-duplex. Thus, the mesh network of $m$ interfaces per node, and $c$ channels will be noted 
as $m, c$-network. Suppose each channel can support a multi-path dependent data rate of $R=R_{\text {multipath }}$, independent of number of non-overlapping channels of the network. Then, the total data rate possible by using all $c$ non-overlapping channels is $R c$. The number of non-overlapping channels can be increased by utilizing extra frequency spectrum of the standard technologies [13].

\subsection{Capacity limit for irregular placement}

Consider Figure 3 that shows irregular placements of HPNs in a fixed area. In rural areas, the inter node distance is large and the topography of landscape affects node placements. To avoid interference, it is assumed that no any two HPNs are placed within a radius less than $400 \mathrm{~m}$ at the edge and less than 700 $\mathrm{m}$ toward the centre of the deployment area. However, between any two HPNs the largest separation distance is allowed as much possible as the size of the area can accommodate. Thus, a capacity theorem can be formulated.

Theorem 1: The E2E upper bound on capacity of a statically assigned channel network of type $m, c$-arbitrary and irregular placement of HPNs is found to be, $\lambda n \bar{L}=O\left(R n \sqrt{\frac{m c}{\delta p}}\right)$ bit-meters/sec when $\frac{c}{m}=O(n)$.

Proof: Let us consider that in irregular and static networks, the node density varies over space (i.e., an area) but stays constant over time. Suppose the regularity rate (probability) of HPN placements is denoted as $0<p<1$, then the area $A$ is defined as $A=n / \delta p$. Thus, the capacity of the network is assumed to be inversely proportional to the $\delta p$ factor for an irregular placement.

Define the capacity of each channel, $R$ as $R=k A=k n / \delta p$ for some constant $k$ (in bits/s/square meters). Suppose each source HPN can generate packets depending on the application at a rate of $\lambda$ bits/sec and the mean separation distance between the source and destination HPN pairs is $\bar{L}$ meters (via multiple hops), then the E2E network capacity of the network is given by [7]:

$$
\lambda n \bar{L}, \text { bit-meters/sec. }
$$

The expression in (1) does not take into account the number of frequency channels, interference, path loss effects and number of interface cards. Relating this high level network capacity with the actual number of hops between the source and destination nodes requires that the overall bits transported in the network be evaluated as follows. Suppose bit $b, 1 \leq b \leq \lambda n$ (bits/sec), traverses $h(b)$ hops on the path from its source to its destination, where the $h^{\text {th }}$ hop traverses a distance of $r_{b}^{h}$, then the overall bits transported in the network in every second is summed. This summation must be at least that in (1):

$$
\lambda n \bar{L} \leq \sum_{b=1}^{\lambda n} \sum_{h=1}^{h(b)} r_{b}^{h}, \text { bit - meters } / \text { sec. }
$$

The inequality in (2) holds since the mean length of the line joining the source and destination, is equal to at most the distance traversed by a bit from its sources to its destination [8]. Additionally, HPNs have $m$ interfaces per node 
and with achievable data rate of $R$ possible per channel. Then, the total bits per second that can be transmitted by all interfaces in the network and all channels is at most $\frac{R n m c}{2}$ (transporting one bit across one hop requires two interfaces, one each at the transmitting and the receiving nodes). Let $X=\sum_{b=1}^{\lambda n} X(b)$ as the number of bits transmitted by all nodes in a second (including bits forwarded). Then, the relation between the rate of a single channel link, the number of interface cards creating single links, the number of nodes in the network, and the total number of hops traversed by all bits in every second is given by,

$$
X \leq \frac{R m n}{2}, \text { bits } / \text { sec }
$$

It should be noted that under the interference protocol model [8], a transmission over a hop of length $r$ in a path loss link is successful only if there can be no active transmitter within a distance of $(1+\triangle) r$. In IEEE $802.11 \mathrm{a} / \mathrm{b} / \mathrm{g} / \mathrm{n}$ standards, the medium access control (MAC) layer protocols execute carrier sense multiple access with collision avoidance (CSMA/CA) mechanism which ensures that this condition is always satisfied. To illustrate this concept further, suppose node $\mathrm{A}$ is transmitting a bit to node $\mathrm{B}$, while node $\mathrm{C}$ on one side of node $\mathrm{B}$, is simultaneously transmitting a bit to node $\mathrm{D}$ furthest from node $\mathrm{A}$ and both the sessions are over a common frequency channel, $\mathrm{W}$. Then, using the interference protocol model and the geometry sufficient for successful reception, node $\mathrm{E}$ which is between nodes $\mathrm{B}$ and $\mathrm{C}$, cannot transmit at the same time with nodes $\mathrm{A}$ and $\mathrm{C}[6]$. That is,

$$
d(C, B) \geq(1+\triangle) d(A, B) \quad \text { and } \quad d(A, D) \geq(1+\triangle) d(C, D) .
$$

Adding the two inequalities together, and applying the triangle inequality to (4), we can obtain the inequality in (5),

$$
d(B, D) \geq \frac{\triangle}{2}(d(A, B)+d(C, D)) .
$$

Therefore, in collision avoidance (CSMA/CA) principle, expression (5) can be viewed as each hop covering a disk of radius $\frac{\Delta}{2}$ times the length of the hop around each receiver. Then, the separation distance between receiver $\mathrm{B}$ and transmitter $\mathrm{C}$ is at least $(A B+\triangle A B)$ and that of transmitter $\mathrm{A}$ and receiver $\mathrm{D}$ is at least $(C D+\triangle C D)$. Thus, the summation over all channels (which can potentially transport $R_{c}$ bits per second) will yield the constraint formulated as:

$$
\sum_{b=1}^{\lambda n} \sum_{h=1}^{h(b)}\left(r_{b}^{h}\right)^{2} \leq A R_{c} \Rightarrow \sum_{b=1}^{\lambda n} \sum_{h=1}^{h(b)} \frac{1}{X}\left(r_{b}^{h}\right)^{2} \leq \frac{4 A R_{c}}{\pi \triangle^{2} X} .
$$

Using the convex inequality rule [6] the left hand side in (6) is rewritten as,

$$
\left(\sum_{b=1}^{\lambda n} \sum_{h=1}^{h(b)} \frac{1}{X} r_{b}^{h}\right)^{2} \leq \sum_{b=1}^{\lambda n} \sum_{h=1}^{h(b)} \frac{1}{X}\left(r_{b}^{h}\right)^{2} .
$$


Therefore, from (6) and (7) one gets,

$$
\sum_{b=1}^{\lambda n} \sum_{h=1}^{h(b)} r_{b}^{h} \leq \sqrt{\frac{4 A R_{c} X}{\pi \triangle^{2}}}
$$

From (8), it can be found that:

$$
\sum_{b=1}^{h n} \sum_{h=1}^{h(b)} \frac{1}{X} r_{b}^{h} \leq \sqrt{\frac{4 A R R_{n m c}}{2 \pi \triangle^{2}}} \Rightarrow \sum_{b=1}^{h n} \sum_{h=1}^{h(b)} r_{b}^{h}=\lambda n \bar{L} .
$$

So that,

$$
\lambda n \bar{L} \leq R \sqrt{\frac{2 A n m c}{\pi \triangle^{2}}}=R n \sqrt{\frac{2 m c}{\delta p \pi \triangle^{2}}} \Rightarrow \lambda n \bar{L}=O\left(R n \sqrt{\frac{m c}{\delta p}}\right), \text { bit-meters } / s .
$$

\subsection{Capacity limit for clustered placement in real network}

Suppose that $n$ nodes are arbitrarily located in a cluster fashion on a square of a fixed area with a guaranteed line of sight (LOS) is guaranteed between any two neighbouring nodes shown in Figure 4. Thus, within a cluster a minimum separation distance of $700 \mathrm{~m}$ is considered, while any largest separation distance possible is considered between clusters. Thus, the following theorem on capacity limit can be formulated.

Theorem 2: The E2E upper bound on capacity of statically a signed channel network of type $m, c$-arbitrary clustered placement of nodes when $\frac{c}{m}=O(n)$ is given as $\lambda n \bar{L}=O\left(R \sqrt{n m c\left(\frac{n_{1}}{\delta_{1}}+\frac{n_{2}}{\delta_{2}}\right)}\right)$ in bit-meters/sec, where $R$ is the $\min (R 1, R 2)$ for intra and inter cluster rates, $n_{1}$ are number of nodes in a regular cluster and $n_{2}$ are number of clusters in the network.

Proof: We assume a clustered placement of the mesh network as a special case of regular or uniform HPNs placement [6]. However, in this case the node densities are: $\delta_{1}=n_{1} / A_{1}$ as the density of nodes within a cluster consisting of $n_{1}$ nodes in $A_{1}$ of an area and as the density of clusters consisting of $n_{2}$ clusters in $A_{2}$ of an area. This assumption is reasonable since HPNs within a cluster form different densities from those between clusters. Thus, the application layer generates the E2E capacity that depends on the number of nodes denoted as $\lambda n \bar{L}$ bit-meters/s. Suppose bit $b, 1 \leq b \leq \lambda n$ (bits/sec), traverses $h(b)$ hops on the path from its source to its destination, where the $h^{\text {th }}$ hop traverses a distance of $r_{b}^{h}=r_{b}^{h}\left(\delta_{1}\right)+r_{b}^{h}\left(\delta_{2}\right)$. That is, the intra-cluster and inter-cluster components of hop distance. Then, one obtains network capacity by summing over all bits in the network as have provided in (2). Therefore, using similar arguments, steps (3), (7) through (10) as provided in the Proof of Theorem 1, the interference constraint protocol holds for any networks [6]. Consequently, the derived E2E capacity limit will be upper bound according to: 


$$
\lambda n \bar{L} \leq R \sqrt{\frac{2 n m c}{\pi \triangle^{2}}\left(\frac{n_{1}}{\delta_{1}}+\frac{n_{2}}{\delta_{2}}\right)}, \text { bit - meter } / \text { sec }
$$

So that the asymptotic E2E upper bound capacity limit for a clustered placement of an HPN network is given by:

$$
\lambda n \bar{L}=O\left(R \sqrt{n m c\left(\frac{n_{1}}{\delta_{1}}+\frac{n_{2}}{\delta_{2}}\right)}\right), \text { bit }- \text { meter } / \text { sec }
$$

In all derivations, $R$ is a dependent variable that varies with the number of multiple paths, number of antennas and antenna gains [6], [15]. The link rate is calculated using models derived in [6].

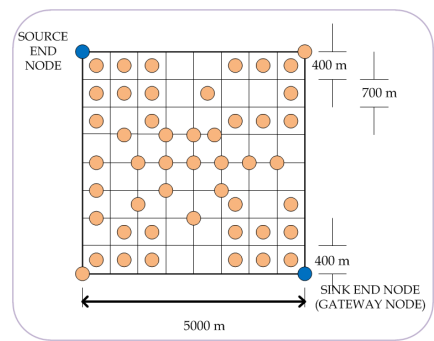

Fig. 3: Irregular placement

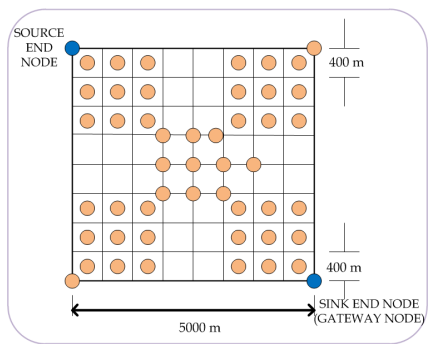

Fig. 4: Clustered placement

\section{Numerical examples}

\subsection{Conditions and results of E2E achievable capacity}

Results in Tables 1,2 and 3 are obtained based on the datasheets of IEEE 802.11a/n standards [13]. Table 1 records the effects of irregular placement on the E2E capacity limits of IEEE 802.11a HPNs. Tables 2 and 3 show the E2E numerical values of achievable capacity computed right from the Ethernet at one end of the network to Ethernet at the other end of the network. The results assume that the interfaces, $m=2$, the channels $c=2$, the deployment area $A=5000 m x 5000 m$, and the bandwidth $W=20 \mathrm{MHz}$ at carrier frequencies $c 1=5.260 \mathrm{GHz}$, tuned to an $8 \mathrm{dBi}$ omni-antenna and $c 2=5.725 \mathrm{GHz}$, tuned to a $20 \mathrm{dBi}$ directional antenna. The OFDM sub channels $=48$, the effective antennae gain $=28 \mathrm{dBi}$ and hilly path loss exponent $=3$ are assumed. Suppose that CSMA/CA protocol is employed in order to identify node pairs that can simultaneously transmit [1]. Also, let $\triangle=10 \%$ defines a fraction of one hop distance which is sufficient to prevent neighboring nodes from transmitting on the same 


\begin{tabular}{|c|c|c|c|c|c|c|c|c|c|c|}
\hline $\mathrm{p}$ & 0.1 & 0.2 & 0.3 & 0.4 & 0.5 & 0.6 & 0.7 & 0.8 & $\begin{array}{l}0.9 \\
\end{array}$ & 1 \\
\hline $\begin{array}{l}\text { E2E } \\
\text { capacity } \\
\text { (Mbps) }\end{array}$ & $\begin{array}{l}0 \\
\text { d. } \\
0 \\
\text { d. }\end{array}$ & $\begin{array}{l}v \\
0 \\
0 \\
i \\
i\end{array}$ & 胥 & 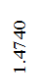 & $\stackrel{\text { d }}{\stackrel{\Phi}{m}}$ & 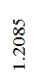 & $\stackrel{-7}{7}$ & స్ & $\begin{array}{l}\text { ô } \\
\text { ox } \\
0 \\
0\end{array}$ & $\begin{array}{l}\text { ב్ల } \\
\text { ô. }\end{array}$ \\
\hline
\end{tabular}

Table 1: Placement Irregulaity versus E2E capacity of IEEE802.11a HPNs

\begin{tabular}{|c|c|c|c|}
\hline $\begin{array}{c}\text { HPNs placement } \\
\text { in a } 5 \mathrm{~km} \times 5 \mathrm{~km} \text { area }\end{array}$ & $\begin{array}{l}\text { No. } \\
\text { HPNs }\end{array}$ & $\begin{array}{c}\begin{array}{c}\text { Achievable link capacity } \\
(M \text { Mps })\end{array} \\
\text { ( }\end{array}$ & $\begin{array}{l}\text { E2E achievable } \\
\text { capacity (Mbps) }\end{array}$ \\
\hline \multirow[t]{2}{*}{$\begin{array}{l}\text { Regular at } p= \\
100 \%\end{array}$} & 10 & $\mathrm{R}(2100 \mathrm{~m})=281.12$ & 0.5192 \\
\hline & 50 & $\mathrm{R}(700 \mathrm{~m})=376.22$ & 0.9322 \\
\hline \multirow{2}{*}{$\begin{array}{l}\text { Irregular at } p= \\
90 \%\end{array}$} & 10 & $\mathrm{R}(2100 \mathrm{~m})=281.12$ & 0.5473 \\
\hline & 50 & $\mathrm{R}(700 \mathrm{~m})=376.22$ & 0.9827 \\
\hline \multirow[t]{2}{*}{ Clustered } & 10 & $\begin{array}{l}\mathrm{R}_{1}(700 \mathrm{~m})=376.22 \\
\mathrm{R}_{2}(4200 \mathrm{~m})=221.13 \\
\mathrm{R}=\min \left(\mathrm{R}_{1}, \mathrm{R}_{2}\right)\end{array}$ & 0.4202 \\
\hline & 50 & $\begin{array}{l}\mathrm{R}_{1}(700 \mathrm{~m})=376.22 \\
\mathrm{R}_{2}(1400 \mathrm{~m})=316.22 \\
\mathrm{R}=\min \left(\mathrm{R}_{1}, \mathrm{R}_{2}\right)\end{array}$ & 0.5374 \\
\hline
\end{tabular}

Table 2: Numerical values of E2E capacity in IEEE 802.11a of HPNs

\begin{tabular}{|c|c|c|c|}
\hline $\begin{array}{c}\text { HPNs placement } \\
\text { in a } 5 \mathrm{~km} \times 5 \mathrm{~km} \text { area }\end{array}$ & $\begin{array}{c}\text { No. of } \\
\text { HPNs }\end{array}$ & $\begin{array}{c}\text { Achievable link capacity } \\
\text { (Mbps) }\end{array}$ & $\begin{array}{l}\text { E2E achievable } \\
\text { capacity (Mbps) }\end{array}$ \\
\hline \multirow[t]{2}{*}{$\begin{array}{l}\text { Regular at } p= \\
100 \%\end{array}$} & 10 & $\mathrm{R}(2100 \mathrm{~m})=722.24$ & 1.3339 \\
\hline & 50 & $R(700 \mathrm{~m})=912.44$ & 2.2609 \\
\hline \multirow[t]{2}{*}{$\begin{array}{l}\text { Irregular at } p= \\
90 \%\end{array}$} & 10 & $\mathrm{R}(2100 \mathrm{~m})=722.24$ & 1.4061 \\
\hline & 50 & $\mathrm{R}(700 \mathrm{~m})=912.44$ & 2.3832 \\
\hline \multirow[t]{2}{*}{ Clustered } & 10 & $\begin{array}{l}\mathrm{R}_{1}(700 \mathrm{~m})=912.44 \\
\mathrm{R}_{2}(4200 \mathrm{~m})=602.24 \\
\mathrm{R}=\min \left(\mathrm{R}_{1}, \mathrm{R}_{2}\right)\end{array}$ & 1.1443 \\
\hline & 50 & $\begin{array}{l}\mathrm{R}_{\mathrm{R}}(700 \mathrm{~m})=912.44 \\
\mathrm{R}_{2}(1400 \mathrm{~m})=792.44 \\
\mathrm{R}=\min \left(\mathrm{R}_{1}, \mathrm{R}_{2}\right)\end{array}$ & 2.0201 \\
\hline
\end{tabular}

Table 3: Numerical values of E2E capacity in IEEE 802.11n of HPNs

sub channel at the same time. If an optimized link state routing (OLSR) protocol that proactively maintains fresh lists of destinations and their routes is also considered [14]. Then, routing tables are periodically distributed in the network. The OLSR ensures that a route to a particular destination is immediately available. The expected transmission count (ETX) metric to calculate the expected number of retransmissions that are required for a packet to travel to and from a destination can be adopted [14]. Using ETX information, the E2E capacity designs can be validated practically. In particular, consider the following cases: irregular pattern when $n=10$ and when $n=50$. Assume that the average distance of source-destination pair is $6505 \mathrm{~m}$. The value enables the computation of achievable capacity over direct LOS path (i.e. without multi-hops) between the source and destination nodes. For irregular placements, the rate (probability) $p$ is constrained by $0<p<1$. The choice of $p$ depicts the degree of irregularity, with smaller values of $p$ depicting more irregular placement. 


\subsection{Discussions on E2E achievable capacity}

From Table 1, the average node density is inversely proportional to the E2E capacity according to Theorem 1 . Thus, a lower average density in an irregular node placement for the same number of nodes will yield a higher E2E capacity if and only if the area of deployment is fixed or decreased. Using similar argument, when values of $p$ is decreased (i.e., $0.8,0.7,0.6$, etc.), the average $\delta$ decreases proportionately and if the area of deployment is fixed or reduced then for the same number of nodes, the capacity will increase. From Tables 2 and 3, in a fixed area of $5 \mathrm{~km}$ by $5 \mathrm{~km}$, the E2E achievable capacity evaluated shows that there is lower capacity when number of HPNs is ten than when the number is 50 for both irregular and clustered placements. The reason is that a series of long links created between any two immediate nodes degrades the achievable E2E capacity, due to long link attenuations. For instance, at ten HPNs in the fixed sized network, the hop distances are much larger than the case for $50 \mathrm{HPNs}$. In each hop, the propagating signal is attenuated by the terrain irregularity, foliage and wireless medium conductivity. The implication is that signal traversing longer hop distances are faced with higher attenuation resulting in lower E2E capacity than signal propagating over shorter hops. Although effects of forwarding delays exacerbate throughput, delays due to lossy links degrades the capacity more. Considering the same number of nodes and fixed area of deployment, the inter hop distances defining the position of the nodes will be much smaller by $10 \%$ than in the case of regular HPN placements when $p=100 \%$. However, shorter hops imply higher capacity if and only if there is limited interference and forwarding delays. Moreover, increasing or keeping constant the number of nodes placed in a fixed area automatically increases or keeps constant the average node density. It was also noted that network throughput dropped significantly from source HPN to the destination HPN or the gateway. In particular, the drop was by about $99 \%$ across 3 long distance hops and by about $99 \%$ across 3 long distance hops considering irregularly and clustered HPNs from Tables 2 and 3, respectively. The explanation is that, the channel gain drops with increase in propagation distance, and there are also overhead losses associated with MAC and the multi-hop routing such that the number of packets sent is not always equal to the number of packets received successfully, even in free space medium. Despite this observation, HPNs derived from IEEE 802.11n radios have a better E2E capacity achievable mainly due to the MIMO technologies that are capable of combating multi-path fading [15].

\section{Conclusions}

The HPNs ${ }^{\mathrm{TM}}$ architecture makes use of omni-directional antennas to maintain mesh connectivity, while directional antennas support information relay over long distances with high power gains. It was confirmed analytically and numerically that increasing the number of interfaces per HPN with carefully configured antennas and non overlapping frequency channels in the network does increase 
the achievable E2E capacity in any arbitral network placement. For overlapping channels, the capacity depends on the dynamic channel assignment and transmit power control which is in the future work. One of the contributions of this study was the design of capacity for the innovation constructed to improve performance of the commercially available WLAN devices. Other possible explorations of increasing capacity of community networks such as Peebles valley mesh in South Africa [6] include the utilization of unused TV frequency spectrum. The low frequencies of the TV spectrum foster high capacity signal transmissions over long distances in rural terrains. Thus, cognitive and energy foraging radio techniques are promising tools toward spectrum and energy efficient network management for the next billion internet users.

\section{References}

1. Kodialam, M., Nandagopal. T.: Characterizing the capacity region in multi-radio multi-channel wireless mesh networks. MobiCom05, Cologne, Germany, (2005).

2. Olwal, T. O.: Decentralised dynamic power control for wireless backbone mesh networks, PhD Thesis, University of Paris-EST and TUT (2010).

3. Olwal, T. O et al.: Optimal control of transmission power management in wireless backbone meshes networks, In: Wireless Mesh Networks, Funabiki, N (Eds). pp. 3-28, InTech, Croatia (2011).

4. Eriksson, J.; Agarwal, S.; Bahl, P. \& Padhye, J.: Feasibility study of mesh networks for all-wireless offices, In Proc. of MobiSys06, Sweden, pp. 69-82, (2006).

5. Mesh Dynamics Inc.: Wireless mesh networks that scale like switch stacks, Available at http://www.meshdynamics.com.(2010).

6. Olwal, T.O., et al.: Achievable capacity limit of high performance nodes for wireless mesh networks. Wireless Mesh Networks: InTech, pp. 1-28, Krendzel (Eds), (2012).

7. Gupta, P., and Kumar, P. R.: The capacity of wireless networks. IEEE Transactions on 4 Information Theory, vol. 46, no. 2, pp. 388-404, (2000).

8. Kyasanur, P. \& Vaidya, N. H.: Capacity of multi-channel wireless networks: impact of number of channels and interfaces, Technical Report, Dep. of Comp. Science, Univ. of Illinois, Urban-Champaign, (2005).

9. Berthilson, L. \& Pascual, A. E.: Link performance parameters in IEEE 802.11: How to increase the throughput of a wireless long distance link, White paper, (2007).

10. Makitla, I. ; Makan, A. \& Roux, K.: Broadband provision to underprivileged rural communities. Proc. of CSIR 3rd Biennial Conference (2010).

11. Ishmael, J., Bury, S., Pezaros, D., \& Race, N.: Deployment rural community wireless mesh networks. IEEE Internet Computing. Vol. (2): 22-29, (2008).

12. Roux, R., et al.: Broadband for all (BB4all) ${ }^{\mathrm{TM}}$, CSIR Science Scope Magazine, vol. 3, no. 3, pp. 16-17 (2009).

13. Cisco Systems Inc.: Channels and maximum power settings for Cisco Aironet lightweight access points. Pp. 1-130, No: OL-11321-08 (2011).

14. Johnson, D.L. \& Roux, K.: Building rural wireless networks: lesions learnt and future directions, Proc. of ACM Workshop on Wireless Networks and Systems for Developing Regions, San Francisco, USA, pp. 17- 22, (2008).

15. Franceschetti, M, et al.: The capacity of wireless networks: information-theoretic and physical limits. IEEE Trans. on Inf. Theory. vol. 55. no. 8, pp. 3413-3424 (2009). 